\title{
X-RAY CRYSTALLOGRAPHIC STRUCTURAL STUDY ON A CINNAMOLIDE-CLASS SESQUITERPENE LACTONE FROM DRIMYS WINTERI FORST. VAR. CHILENSIS
}

\author{
IVÁN BRITO*I, ALEJANDRO CÁRDENAS ${ }^{2}$, MIGUEL ZÁRRAGA3 , CRISTIÁN PAZZ , CLAUDIA PÉREZ4 AND \\ MATÍAS LÓPEZ-RODRÍGUEZ
}

\begin{abstract}
${ }^{1}$ Departamento de Química, Facultad de Ciencias Básicas, Universidad de Antofagasta, Casilla 170, Antofagasta-Chile.
${ }^{2}$ Departamento de Física, Facultad de Ciencias Básicas, Universidad de Antofagasta, Casilla 170, Antofagasta-Chile.

${ }^{3}$ Departamento de Química Orgánica, Facultad de Ciencias Químicas, Universidad de Concepción, Casilla 160-C, Concepción-Chile.

${ }^{4}$ Laboratorio de Fitoquímica, Facultad de Ciencias Biológicas, Universidad de Concepción y Centro de Investigación de Ecosistemas de la Patagonia (CIEP). Bilbao 449, Coyhaique-Chile.

${ }^{5}$ Instituto de Bio-Orgánica 'Antonio González', Universidad de La Laguna, Astrofisico Francisco Sánchez $N^{\circ}$ 2, La Laguna, Tenerife, España.
\end{abstract} (Received: April 25, 2008 - Accepted: August 1, 2008)

\begin{abstract}
In order to ascertain the structure and secure the assignment of the stereochemistry of the title compound an X-ray analysis was performed, the absolute configuration was not determined by this analysis.
\end{abstract}

Keywords: X-ray diffraction, cinnamolide - class sesquiterpene.

\section{INTRODUCTION}

Drimys winteri J.R. Forst is a plant used in folk medicine of the many Latinoamerican countries. In Chile, Drimys winteri (canelo) is used by the indigenous Mapuche in the treatment of several stomachal diseases, ulcers and hemorrhages ${ }^{1}$. Chemical studies has shown the presence of a variety of sesquiterpenes with drimano skeleton ${ }^{2}$ and flavonoids. Some of these structures have shown significant antibacterial, antifungi, antitumor and insecticide properties ${ }^{3,4}$. The extract of Drimys winteri leaves afforded Cinnamolide and Drimenin two lactones with drimano skeleton. Cinnamolide have not been previuosly reported for these specie. Recently it has been reported that the title compound was also isolated of Cinnamosma macrocarpa ${ }^{5}$. In order to ascertain the structure and secure the assignment of the stereochemistry of the title compound an X-ray analysis was performed, the absolute configuration was not determined by this analysis. The X-ray crystallographic analysis confirms the previously proposed molecular structure, including the molecular conformation. The molecular structure is shown in Fig.1 and selected geometric parameters are given in Table I. The structure consists of a drimane skeleton.
The methyl group at $\mathrm{C} 9 \mathrm{a}$ is seen to be $\beta$-oriented. The cyclohexene ring A and cyclohexane ring $\mathrm{B}$ exist in a half-chair and a chair conformation, respectively $\left[\mathrm{Q}_{\mathrm{T}}=0.538(3) \AA, \varphi_{2}=227.4(4)^{\circ}, \mathrm{q}_{2}=0.428\right.$ (3) $\AA$ for ring $\mathrm{A} ; \mathrm{Q}_{\mathrm{T}}=0.556(4) \AA$ $, \varphi_{2}=21(4)^{\circ}, q_{2}=0.060$ (4) $\AA$ for ring $\left.B\right]$, and the lactone ring an envelope on $\mathrm{C} 3$ a conformation ${ }^{6}$. The $\mathrm{A}$ and $\mathrm{B}$ rings are trans-fused. Bond lengths are within expected ranges ${ }^{7}$. There are no unusually short contacts between the molecules and the crystal packing is controlled by weak van der Waals interactions.

\section{Source of material}

Drimys winteri was collected from the Estuary of Reloncaví, $X^{\text {th }}$ Región, Chile in November 2005. $2 \mathrm{~kg}$ of bark was extracted in dichloromethane and concentrated under vacuum, to yield 180 g. 30 grams of crude extract was subjected to flash chromatography on silicagel G, 70-200 mesh with hexane-ethyl acetate mixtures of increasing polarity as elution solvents. Pure components were obtained by further chromatography on silicagel of the fraction $10 \%$ hexane-ethyl acetate $(11 \mathrm{~g})$ and recrystallization from methanol, at room temperature afforded colorless crystals of Drimenin $(0.02 \mathrm{~g})$ suitable for X-ray diffraction analysis.

Table I. Structure determination summary for Drimenin $\left(\mathrm{C}_{15} \mathrm{H}_{22} \mathrm{O}_{2}\right)$.

\begin{tabular}{|c|c|}
\hline Wavelength & $0.71073 \AA$ \\
\hline Crystal system & Orthorhombic \\
\hline Space group & $\mathrm{P} 22_{1} 2_{1}$ \\
\hline \multirow[t]{3}{*}{ Unit cell dimensions } & $\mathrm{a}=7.6271(11) \AA$ \\
\hline & $\mathrm{b}=12.0631(9) \AA$ \\
\hline & $\mathrm{c}=14.5232(13) \AA$ \\
\hline Crystal & Colorless, prism, $0.13 \times 0.10 \times 0.08 \mathrm{~mm}$ \\
\hline Volume, Z & $1336.2(2) \AA^{3}, 4$ \\
\hline Absorption coefficient & $0.075 \mathrm{~mm}^{-1}$ \\
\hline Diffractometer, scan mode & Nonius KappaCCD area-detector diffractometer, $\omega / 2 \theta$ \\
\hline $\mathrm{N}(\mathrm{hkl}$ )measured,N(hkl)unique & 4398,1692 \\
\hline Reflections observed, $\mathrm{I}>2 \sigma(\mathrm{I})$ & 1444 \\
\hline Final $\mathrm{R}$ indices & $\mathrm{R}_{1}=0.0601 \quad \mathrm{wR}_{2}=0.1380$ \\
\hline $\mathrm{R}$ indices (all data) & $\mathrm{R}_{1}=0.0749 \quad \mathrm{wR}_{2}=0.1503$ \\
\hline Largest diff. peak and hole & 0.202 and -0.149 e. $\AA^{-3}$ \\
\hline Computing structure solution & SIR97 ${ }^{8}$ \\
\hline Computing structure refinement & SHELXL-97 ${ }^{9}$ \\
\hline Refine 1s. hydrogen treatment & Treated by a mixture of independent and constrained refinement \\
\hline
\end{tabular}


Table II. Atomic coordinates $\left(\AA \times 10^{4}\right)$ and equivalent isotropic displacement parameters $\left(\AA^{2} \times 10^{3}\right) . \mathrm{U}(\mathrm{eq})=(1 / 3) \sum_{\mathrm{i}} \Sigma_{\mathrm{j}} \mathrm{U}_{\mathrm{ij}} \mathrm{a}_{\mathrm{i}} * \mathrm{a}_{\mathrm{j}} * \mathrm{a}_{\mathrm{i}} \mathrm{a}_{\mathrm{j}}$ founded for $\left(\mathrm{C}_{15} \mathrm{H}_{22} \mathrm{O}_{2}\right)$.

\begin{tabular}{|l|l|l|l|l|}
\hline \multicolumn{1}{|c|}{ Atom } & $\mathrm{x}$ & $\mathrm{y}$ & $\mathrm{z}$ & $\mathrm{U}(\mathrm{eq})$ \\
\hline & & & & \\
\hline $\mathrm{O}(1)$ & $6371(4)$ & $2125(3)$ & $8176(2)$ & $87(1)$ \\
\hline $\mathrm{O}(2)$ & $5174(4)$ & $1983(2)$ & $9565(2)$ & $66(1)$ \\
\hline $\mathrm{C}(1)$ & $3742(5)$ & $1387(3)$ & $10002(2)$ & $60(1)$ \\
\hline $\mathrm{C}(3)$ & $5208(5)$ & $1779(3)$ & $8655(3)$ & $56(1)$ \\
\hline $\mathrm{C}(3 \mathrm{~A})$ & $3667(4)$ & $1098(2)$ & $8409(2)$ & $40(1)$ \\
\hline $\mathrm{C}(4)$ & $3468(4)$ & $506(3)$ & $7662(2)$ & $49(1)$ \\
\hline $\mathrm{C}(5)$ & $1943(4)$ & $-261(3)$ & $7539(2)$ & $52(1)$ \\
\hline $\mathrm{C}(5 \mathrm{~A})$ & $592(3)$ & $-188(2)$ & $8330(2)$ & $35(1)$ \\
\hline $\mathrm{C}(6)$ & $-866(4)$ & $-1100(3)$ & $8262(3)$ & $51(1)$ \\
\hline $\mathrm{C}(7)$ & $-2127(5)$ & $-952(3)$ & $9076(3)$ & $63(1)$ \\
\hline $\mathrm{C}(8)$ & $-1253(6)$ & $-903(3)$ & $10006(3)$ & $71(1)$ \\
\hline $\mathrm{C}(9)$ & $52(5)$ & $60(3)$ & $10032(2)$ & $58(1)$ \\
\hline $\mathrm{C}(9 \mathrm{~A})$ & $1483(3)$ & $-55(2)$ & $9285(2)$ & $35(1)$ \\
\hline $\mathrm{C}(9 \mathrm{~B})$ & $2480(4)$ & $1059(2)$ & $9223(2)$ & $38(1)$ \\
\hline $\mathrm{C}(10)$ & $2759(5)$ & $-1003(3)$ & $9508(3)$ & $59(1)$ \\
\hline $\mathrm{C}(11)$ & $-196(5)$ & $-2307(3)$ & $8245(4)$ & $75(1)$ \\
\hline $\mathrm{C}(12)$ & $-1907(6)$ & $-893(4)$ & $7366(3)$ & $81(1)$ \\
\hline & & & & \\
\hline
\end{tabular}

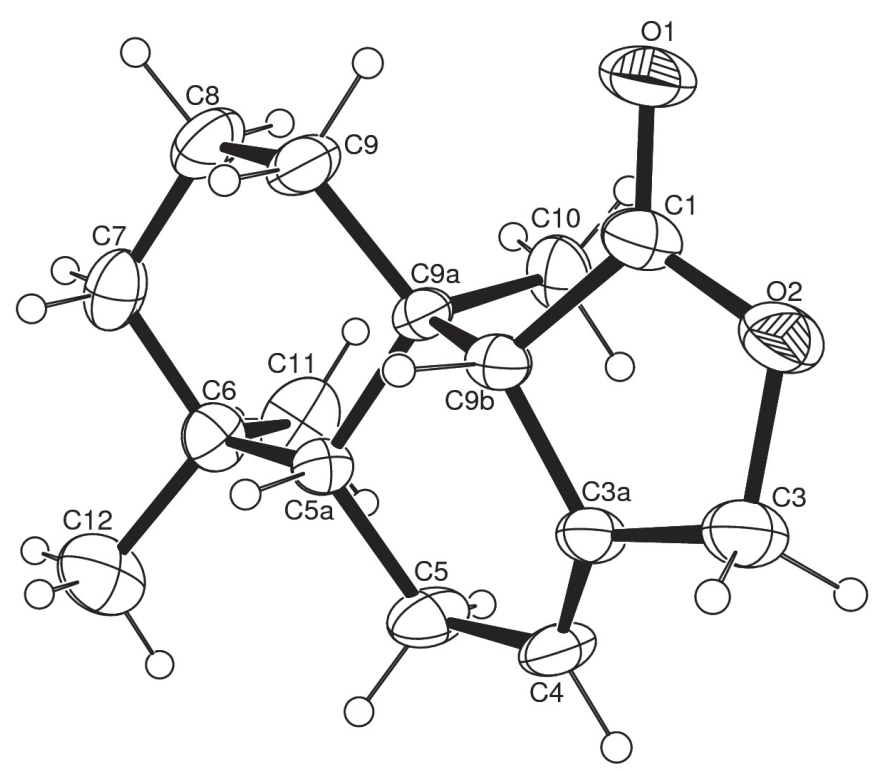

Figure 1. A view of the molecular structure of $\left(\mathrm{C}_{15} \mathrm{H}_{22} \mathrm{O}_{2}\right)$, showing the atom-numbering scheme. Displacement ellipsoids are drawn at the $30 \%$ probability level and $\mathrm{H}$ atoms are shown as small spheres of arbitrary radii (ORTEP-3) ${ }^{10}$

\section{ACKNOWLEDGEMENTS}

We thank the Spanish Research Council (CSIC) for providing us with a free-of-charge licence for the CSD system. M. Z. recognises support provided by the Center for Ecosystem Research in Patagonia (CIEP), under grant 205.023.040-1SP.

\section{REFERENCES}

1. A. T. Almeida, R. Lacerda \& H. Kloos. Mem. Inst. Oswaldo Cruz, 831, (2001)

2. H. H. Appel, R. P. M. Bond \& K. H. Overton..Tetrahedron, 19, 635, (1963)

3. A. Cruz, M. Silva \& P. G. Sammmes. Phytochemistry, 12, 2549, (1973)

4. J. Sierra, J. López, \& M. Cortés. Phytochemistry, 25, 253. (1986).

5. L. Harinanteaina, Y. Asakawa \& E. De Clercq. J. Nat. Prod. 70, 277, (2007)

6. D. Cremer \& J. A. Pople. J. Am. Chem. Soc. 97, 1354, (1975)

7. F. H. Allen, O. Kennard, D. G. Watson, L. Brammer, A. G. Orpen \& R. Taylor. J. Chem. Soc. Perkin Trans. 2, pp. S1-19, (1987)

8. A. Altomare, M. C. Burla, M. Camalli, G. L. Cascarano, C. Giacovazzo, A. Guagliardi, A. G. G. Moliterni, G. Polidori \& R. Spagna. J. Appl. Cryst. 32, 115, (1999).

9. G. M. Sheldrick. Acta Cryst. A64, 112, (2008)

10. L. J. Farrugia. ORTEP-3 for Windows. J. Appl. Cryst. 30, 565. (1997) 